\title{
Befunderhebungsfehler des Radiologen im Mammographie-Screening - aktuelle Rechtsprechung
}

\section{Einführung}

Einer unserer früheren Beiträge behandelte bereits Haftungsfragen im Rahmen des Mammographie-Screenings (RöFo 8/2017, S. 786790). Abzugrenzen ist hierbei insbesondere der Diagnosefehler von dem Befunderhebungsfehler, weil der Diagnosefehler zu einer Haftungsprivilegierung führt, während bei Vorliegen eines Befunderhebungsfehlers Besonderheiten im Rahmen des Beweisrechts gelten (vgl. §630 h Abs. 5 S. 2 BGB).

Ein Diagnosefehler liegt nach ständiger Rechtsprechung des Bundesgerichtshofs $(\mathrm{BGH})$ vor, wenn der Behandler einen tatsächlich erhobenen oder sonst vorliegenden Befund vorwerfbar falsch interpretiert und deshalb (kausal) nicht die aus der berufsfachlichen Sicht seines Fachgebietes gebotenen therapeutischen oder diagnostischen Maßnahmen ergriffen hat $(B G H$, Urt. v. 21.12.2010, Az.: VI ZR 284/09; BGH, Urt. v. 26.01.2016, Az.: VI ZR 146/14; OLG München, Urt. v. 08.08.2013, Az.: 1 U 4549/12). Ein Befunderhebungsfehler liegt hingegen vor, wenn nach dem Stand der medizinischen Wissenschaft gebotene Befunde erst gar nicht erhoben wurden.

Wird ein Patient an einen Radiologen zur Leistungserbringung überwiesen (sog. horizontale Arbeitsteilung), so darf sich der Radiologe grundsätzlich darauf verlassen, dass der überweisende Kollege des anderen Fachgebietes den Patienten in seinem Verantwortungsbereich mit der gebotenen Sorgfalt untersucht und behandelt hat und dass die Indikation zu der erbetenen radiologischen Leistung zutreffend gestellt ist, solange keine offensichtlichen Qualifikationsmängel oder Fehlleistungen des Überweisers erkennbar werden (BGH, Urt. v. 05.10.1993, Az.: VI ZR 237/92). In diesem Sinne urteilte das OLG Hamm (26.05.2005, Az.: 3 U 127/02), dass bei Überweisung einer Patientin vom Gynäkologen zum Radiologen wegen Brustkrebsverdachts ausschließlich zwecks Durchführung einer Mammographie der Radiologe nicht zur einer umfassenden Beratung und Behandlung der Patientin verpflichtet ist.
Es schließt sich jedoch die Frage an, welcher Behandlungsfehlertyp anzunehmen ist, wenn die fehlerhafte Diagnose zur Folge hat, dass keine weiteren Befunde erhoben werden. Ein Diagnosefehler setzt voraus, dass der Behandler die medizinisch notwendigen Befunde überhaupt erhoben hat, um sich eine ausreichende Basis für die Einordnung der Krankheitssymptome zu verschaffen. Hat dagegen die unrichtige diagnostische Einstufung einer Erkrankung ihren Grund bereits darin, dass der Behandler die nach dem medizinischen Standard gebotenen Untersuchungen erst gar nicht veranlasst hat - er mithin aufgrund unzureichender Untersuchungen vorschnell zu einer Diagnose gelangt, ohne diese durch die medizinisch gebotenen Befunderhebungen abzuklären -, dann ist dem Arzt ein Befunderhebungsfehler vorzuwerfen. Rechtlich problematisch an dieser Abgrenzung ist der Umstand, dass die - vorschnelle - Fehldiagnose (= Diagnosefehler) beweisrechtlich „belohnt“ wird, wohingegen dokumentierte Unsicherheiten in der differenzialdiagnostischen Zuordnung (= Befunderhebungsfehler) „bestraft“ werden. Die Lösung dieses Konflikts ist in der Literatur umstritten und war gerichtlich noch nicht explizit entschieden. Ebenfalls nicht abschließend entschieden war der Fall, ob es einen Diagnosefehler darstellt, wenn sich der Arzt in objektiv zweifelhaften Fällen vor endgültiger Diagnosestellung nicht der Richtigkeit seines $\mathrm{Er}$ gebnisses durch Einholung einer zweiten Meinung eines Kollegen versichert. Vor diesem Hintergrund stellte sich die Frage, welche Anforderungen an die Durchführung einer Mammographie-Screening-Untersuchung zu stellen sind; insbesondere, ob die fehlende körperliche Untersuchung der Patientin durch den programmverantwortlichen Arzt (PVA) bei unklarem Befund den Vorwurf eines Befunderhebungsfehlers rechtfertigt.

\section{Urteil des BGH vom 26.05.2020}

Der BGH hat mit Urteil vom 26.05.2020 (Az.: VI ZR 213/19) eine Entscheidung bestätigt, mit der das Landgericht Heilbronn

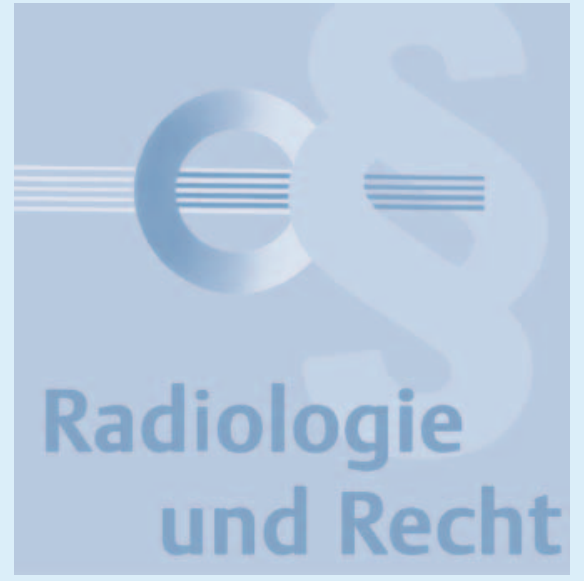

(Urt.v. 19.01.2017, Az.: Ri 1 O 20/15) zwei PVAs einer radiologischen Berufsausübungsgemeinschaft im Zusammenhang mit einem Mammographie-Screening nach der Anlage 9.2 BMV-Ä zur Zahlung von Schmerzensgeld und Ersatz des materiellen Schadens verurteilt und die Ersatzpflicht für künftige Schäden festgestellt hat.

\section{Sachverhalt}

Der Entscheidung liegt folgender Sachverhalt zugrunde: die Patientin unterzog sich Anfang 2010 in der radiologischen Praxis einem Mammographie-Screening - als Ergebnis wurde ihr ein unauffälliger Status mitgeteilt. Nach Krebsvorsorgeuntersuchungen, zuletzt bei Frau Dr. K. am 26.01.2012, bei denen keine pathologischen Befunde festgestellt worden waren, stellte sich die Patientin am 17.04.2012 erneut bei der Praxis zum MammographieScreening vor. In der Anamnese gab sie an, die Mamille rechts sei seit ca. einem Jahr leicht eingezogen. Die Mammographie wurde mit BIRADS 1 (Normalbefund) bewertet; der Patientin wurde mit Schreiben vom 24.04.2012 mitgeteilt, es seien keine Auffälligkeiten festgestellt worden.

Ende April 2014 stellte sich die Patientin bei einem Frauenarzt wegen einer zunehmenden Einziehung der rechten Mamille nebst „Delligkeit“ vor. Die darauffolgenden Untersuchungen führten zur Diagnose von Brustkrebs. Es wurden im weiteren Verlauf ein invasives Karzinom und ein begleitendes lobuläres Karzinom entfernt sowie eine Sentinel-Lymphonodektomie und eine Axilladissektion durchgeführt; in zwei der entnommenen Lymphknoten wurden Metastasen festgestellt. Verdächtiges Gewebe wurde 
nachreseziert, es folgten Bestrahlungen und eine Chemotherapie.

Die Patientin hat geltend gemacht, beide Mammographie-Screenings seien fehlerhaft bewertet und erforderliche weitere Befunderhebungen unterlassen worden. Bei korrektem Vorgehen wäre der Brustkrebs in einem Stadium entdeckt und behandelt worden, in dem noch keine Lymphknoten befallen gewesen wären. Einer Chemotherapie hätte es dann nicht bedurft und die Anzahl der erforderlichen Bestrahlungen wäre geringer gewesen.

\section{Begründung}

Der BGH hat entschieden, dass die Behandlung fehlerhaft war (a.). Da die Behandlung vorliegend nicht in horizontaler Arbeitsteilung erfolgte, konnten die Radiologen nicht auf die sorgfältige Aufgabenerfüllung der mitbehandelnden Frauenärzte vertrauen (b.). Den Behandlungsfehler hat der BGH als Befunderhebungsfehler eingestuft (c.).

\section{a. Behandlungsfehler}

Nach dem BGH liegt ein Behandlungsfehler im Zusammenhang mit dem zweiten Mammographie-Screening vom 17.04.2012 vor, weil die Radiologen es unterlassen haben, der Patientin weitere Untersuchungen zur Abklärung der von ihr geschilderten Mamillenretraktion anzuraten.

Die Radiologen seien zur ordnungsgemäßen Durchführung des MammographieScreenings und zur sorgfältigen Befundung unter Einbeziehung der im Rahmen der Anamnese gewonnenen Erkenntnisse verpflichtet gewesen. Es habe nicht der ärztlichen Sorgfaltspflicht entsprochen, dass sie im Hinblick auf die im Rahmen der Anamnese mitgeteilte Mamillenretraktion nichts weiter unternahmen und der Patientin mit Schreiben vom 24.04.2012 mitteilten, es seien keine Auffälligkeiten festgestellt worden.

Ein Arzt müsse bei einer Beobachtung, die er im Rahmen seiner Untersuchung macht und die auf eine ernst zu nehmende Erkrankung hinweisen kann, auf eine rasche diagnostische Abklärung hinwirken, um vermeidbare Schädigungen des Patienten auszuschließen (BGH, Urt. v. 14.07.1992, Az.: VI ZR 214/91). Er dürfe Auffälligkeiten, die ihm zur Kenntnis gelangen, nicht einfach übergehen. Sogar vor „Zufallsbefunden“, auch solchen, die aus medizinisch nicht gebotenen, aber dennoch veranlassten Untersuchungen herrühren, dürfe er nicht die Augen verschließen (BGH, Urt. v. 21.12.2010, Az.: VI ZR 284/09). So habe der $\mathrm{BGH}$ in dem vorgenannten Urteil entschieden, dass der für die Auswertung eines Befundes verantwortliche Arzt all die Auffälligkeiten zur Kenntnis und zum Anlass für die gebotenen Maßnahmen zu nehmen habe, die er aus berufsfachlicher Sicht seines Fachbereichs unter Berücksichtigung der in seinem Fachbereich vorausgesetzten Kenntnisse und Fähigkeiten sowie der Behandlungssituation feststellen muss.

Im kurativen Bereich der Mammadiagnostik müsse eine Mamillenretraktion abgeklärt werden. Aber auch im Screening-Bereich der Mammadiagnostik zur Krebsfrüherkennung bei diesbezüglichen Erhebungen in der Anamnese und angesichts der vorauszusetzenden Kenntnisse der in diesem Bereich tätigen Ärzte dürfe sie nicht ignoriert werden.

\section{b. Horizontale Arbeitsteilung}

Nach dem Grundsatz der horizontalen Arbeitsteilung habe jeder Arzt denjenigen Gefahren zu begegnen, die in seinem Aufgabenbereich entstehen; er müsse sich aber, jedenfalls solange keine offensichtlichen Qualifikationsmängel oder Fehlleistungen erkennbar werden, darauf verlassen dürfen, dass auch der Kollege des anderen Fachgebiets seine Aufgaben mit der gebotenen Sorgfalt erfüllt. Eine gegenseitige Überwachungspflicht bestehe insoweit nicht (BGH, Urt.v. 26.02.1991, Az.: VI ZR 344/89, Urt. vom 26.01.1999, Az.: VI ZR 376/97).

Die Anwendung dieses Grundsatzes setze schon begrifflich eine Arbeitsteilung, also ein Zusammenwirken von 2 oder mehr Ärzten verschiedener Fachrichtungen voraus (vgl. BGH, Urt. v. 26.01.1999, Az.: VI ZR 376/97; Frahm/Nixdorf, Arzthaftungsrecht, 6. Aufl., Rn. 106). Das Vertrauen in die sorgfältige Aufgabenerfüllung durch den anderen Arzt könne denknotwendig nur begründet werden, wenn ein Arzt von der konkreten Behandlung durch den anderen Arzt Kenntnis hat. Zudem gelte der Vertrauensgrundsatz nur in solchen Konstellationen, in denen es um Gefahren geht, die ausschließlich dem Aufgaben- und Verantwortungsbereich eines der beteiligten Ärzte zugeordnet sind, die Schädigung des Patienten also in einem abgrenzbaren und auf das betreffende Fachgebiet beschränkten Gefahrenkreis eintritt (vgl. BGH, Urt. v. 26.01.1999, Az.: VI ZR 376/97).

Die genannten Voraussetzungen einer horizontalen Arbeitsteilung seien vorliegend nicht erfüllt. Es habe zum Zeitpunkt des Mammographie-Screenings am 17.04.2012 schon an einem irgendwie gearteten Zusammenwirken der Radiologen mit den die Patientin behandelnden Frauenärzten, etwa mit Frau Dr. K., die die letzte Brustkrebsvorsorgeuntersuchung im Januar 2012 vorgenommen hatte, gefehlt. Nach dem Einladungsschreiben zur Mammographie handle es sich bei dem Mammographie-Screening-Programm um eine regelmäßige, alle 2 Jahre durchgeführte Röntgenuntersuchung zur Früherkennung von Brustkrebs. Die Einladung erfolge auf Basis der Melderegisterdaten der Gemeinde (also nicht auf Veranlassung des behandelnden Frauenarztes) und richte sich an Frauen zwischen 50 und 69 Jahren. Das Screening erfolge demnach unabhängig davon, ob und wann zuletzt eine Brustkrebsvorsorgeuntersuchung durch den behandelnden Frauenarzt erfolgt ist. Ein Zusammenwirken der die Mammographie durchführenden Ärzte und des behandelnden Frauenarztes finde daher ohne Hinzutreten weiterer Umstände nicht statt. Eine horizontale Arbeitsteilung werde nicht schon allein dadurch begründet, dass in dem Einladungsschreiben zur Mammographie darauf hingewiesen wird, dass das Screening nur ein „zusätzliches Angebot“ ist und nicht die jährliche Brustkrebsfrüherkennungsuntersuchung beim Frauenarzt ersetzt und dass es für Frauen gedacht ist, bei denen keine Anzeichen für eine Erkrankung der Brust bestehen.

Hinzu komme, dass die Früherkennung des Brustkrebsrisikos in den Aufgabenbereich sowohl der Radiologen als auch der die Patientin behandelnden Frauenärzte fiel, mögen die diesbezüglichen Untersuchungen auch unterschiedlich ausgestaltet gewesen sein. Die Gefahr, im Rahmen der Mammadiagnostik mögliche Anzeichen für Brustkrebs zu übersehen oder ihnen nicht 
nachzugehen, sei also sowohl dem kurativen Bereich als auch dem Bereich des Screenings zuzuordnen.

\section{c. Befunderhebungsfehler}

Ein Befunderhebungsfehler sei gegeben, wenn die Erhebung medizinisch gebotener Befunde unterlassen oder nicht veranlasst wird. Im Unterschied dazu liege ein Diagnoseirrtum vor, wenn der Arzt erhobene oder sonst vorliegende Befunde falsch interpretiert und deshalb nicht die aus der berufsfachlichen Sicht seines Fachbereichs gebotenen - therapeutischen oder diagnostischen - Maßnahmen ergreift (vgl. BGH, Urt. v. 26.01.2016, Az.: VI ZR 146/14, Urt.v. 21.12.2010, Az.: VI ZR 284/09; jeweils m. w. N.). Ein Diagnoseirrtum setze aber voraus, dass der Arzt die medizinisch notwendigen Befunde überhaupt erhoben hat, um sich eine ausreichende Basis für die Einordnung der Krankheitssymptome zu verschaffen. Habe dagegen die unrichtige diagnostische Einstufung einer Erkrankung ihren Grund bereits darin, dass der Arzt die nach dem medizinischen Standard gebotenen Untersuchungen erst gar nicht veranlasst hat, er mithin aufgrund unzureichender Untersuchungen vorschnell zu einer Diagnose gelangt, ohne diese durch die medizinisch gebotenen Befunderhebungen abzuklären, dann sei dem Arzt ein Befunderhebungsfehler vorzuwerfen. Denn bei einer solchen Sachlage gehe es im Kern nicht um die Fehlinterpretation von Befunden, sondern um deren Nichterhebung (BGH, Urt.v. 26.01.2016, Az.: VI ZR 146/14 m. w. N.).

Es liege auch kein Fehler der therapeutischen Aufklärung vor. Es sei danach zu differenzieren, ob der Schwerpunkt der Vorwerfbarkeit ärztlichen Fehlverhaltens in der unterbliebenen Befunderhebung als solcher oder in dem Unterlassen von Warnhinweisen zum Zwecke der Sicherstellung des Behandlungserfolgs liegt (BGH, Urt. v. 17.11.2015, Az.: VI ZR 476/14, Urt. v. 11.04.2017, Az.: VI ZR 576/15). Vorliegend fehle es schon an dem Hinweis, dass die in der Anamnese angegebene Mamillenretraktion - auch nach dem Screening - noch kontrollbedürftig ist und dass weitere Untersuchungen zur Abklärung medizinisch geboten sind. Damit liege der Schwerpunkt der Vorwerfbarkeit des ärztlichen Fehlverhaltens hier in der unterbliebenen Befunderhebung. Die Radiologen hätten nicht davon ausgehen dürfen, dass die notwendigen Untersuchungen bereits im Rahmen der jährlichen Brustkrebsvorsorgeuntersuchung erfolgt waren. Hierfür fehle es, wie dargelegt, an der notwendigen Vertrauensgrundlage.

Es spiele für die Qualifizierung des vorliegenden Behandlungsfehlers als Befunderhebungsfehler keine Rolle, ob die Radiologen die weiteren Untersuchungen selbst durchzuführen oder diese der Patientin anzuraten hatten.

Schließlich sei davon auszugehen, dass die Patientin bei dem gebotenen Hinweis, dass die Mamillenretraktion weiterer Abklärung bedarf, eine solche Abklärung veranlasst hätte. Die Beweislast hinsichtlich des Kausalzusammenhangs zwischen der unterbliebenen weiteren Abklärung der Mamillenretraktion und dem zu Axilladissektion und Chemotherapie führenden Lymphknotenbefall treffe die Radiologen. Ein einfacher Befunderhebungsfehler könne zu einer Umkehr der Beweislast hinsichtlich der Kausalität des Behandlungsfehlers für den eingetretenen Gesundheitsschaden führen, wenn sich bei der gebotenen Abklärung der Symptome mit hinreichender Wahrscheinlichkeit ein so deutlicher und gravierender Befund ergeben hätte, dass sich dessen Verkennung als fundamental oder die Nichtreaktion hierauf als grob fehlerhaft darstellen würde und diese Fehler generell geeignet sind, den tatsächlich eingetretenen Gesundheitsschaden herbeizuführen (vgl. nur BGB, Urt. v. 17.11.2015, Az.: VI ZR 476/14 m. w. N.). Diese Voraussetzungen seien vorliegend erfüllt.

\section{Entscheidungen der Vorinstanzen}

Die Auswertung der vorinstanzlichen Entscheidungen des LG Heilbronn und des OLG Stuttgart (Urt. v. 07.05.2019, Az.: 1 U 16/17) offenbart zwei Besonderheiten, welche für die richtige Einordnung des Urteils des $\mathrm{BGH}$ von Bedeutung erscheinen.

Zunächst lässt sich beiden vorinstanzlichen Entscheidungen nicht entnehmen, ob die Radiologen die Angaben auf dem Anamnesebogen tatsächlich wahrgenommen haben oder nicht. Demnach ist unklar, ob die Radiologen die Anamnese zur Kenntnis genommen, aber für ihrerseits nicht handlungsbedürftig eingeschätzt haben oder ob ihnen die Anamnese tatsächlich unbekannt war. Die Kenntnis dieses Hintergrundes ist Voraussetzung für die Beantwortung der Frage, ob und wie der darauffolgende Behandlungsfehler vermieden werden kann.

Zudem hat das OLG Stuttgart das Abschlussschreiben der Radiologen auszugsweise wie folgt wiedergegeben: „Wir freuen uns, Ihnen mitteilen zu können, dass bei Ihrer Mammographie keine Auffälligkeiten festgestellt wurden. Die Begutachtung Ihrer Röntgenaufnahmen ist durch zwei qualifizierte Ärzte erfolgt. [...] Ein großer Teil bedenklicher Veränderungen der Brust kann durch eine Mammographie entdeckt werden. Es gibt jedoch Fälle, in denen solche Veränderungen auf einer Röntgenaufnahme nicht zusehen sind. Sollten Ihnen daher innerhalb der nächsten zwei Jahre Veränderungen an Ihrer Brust auffallen, die Sie besorgen, wenden Sie sich bitte umgehend an einen Arzt Ihres Vertrauens. Nehmen Sie auch zusätzlich zum Mammographie-Screening regelmäßig an den üblichen Vorsorgeuntersuchungen Ihres Frauen- und Hausarztes teil. "Nach Auffassung des OLG konnte die Patientin dieses Schreiben nur so verstehen, dass die von ihr mitgeteilte Retraktion keinen Anlass zu weiterer Abklärung gab und nur bei neu auftretenden Veränderungen bzw. zur jährlichen Vorsorge ein Arzt konsultiert werden sollte. Genau dies war allerdings unzutreffend. Wiederum zur Fehlervermeidung kommt es darauf an, wie das Schreiben formuliert war und vorwerfbar einen irrtümlichen Eindruck vermitteln konnte.

\section{Folgen des Urteils}

Das Urteil des BGH ist aus der Sicht der betroffenen Patientin zu sehen. Diese ist sich im Regelfall, auch trotz Aufklärung, der begrenzten Aussagekraft des ScreeningBefundes nicht immer umfänglich bewusst; in jedem Fall zumindest grundsätzlich in geringerem Maße als der Radiologe.

Durch das Urteil beantwortet der BGH die im vorangegangenen und eingangs zusammengefassten Beitrag aufgeworfenen Fragen teilweise: die fehlende Behandlung - nicht zwangsläufig (eigene) körperliche Untersuchung - der Patientin durch den PVA bei unklarem Befund kann den Vorwurf eines Befunderhebungsfehlers rechtfertigen. Dies 
gilt jedenfalls dann, wenn die Anamnese Verdachtsmomente begründet, die durch den Befund des Screenings nicht ausgeräumt werden können. Geboten war in diesem Fall zumindest der Hinweis, dass die Mamillenretraktion weiterer Abklärung bedarf.

Nach dem BGH unterfällt das Mammographie-Screening im Regelfall nicht einer horizontalen Arbeitsteilung mit Gynäkologen. In der Folge darf sich ein Radiologe ohne weitere Vergewisserung nicht darauf verlassen, dass auch der gynäkologische Kollege seine Aufgaben mit der gebotenen Sorgfalt erfüllt. Zu berücksichtigen ist dabei, dass die Früherkennung des Brustkrebsrisikos in den Aufgabenbereich sowohl der Radiologen als auch der Gynäkologen fällt.

Die zuvor genannten Besonderheiten aus den vorinstanzlichen Entscheidungen dürften zu einer gewissen Einschränkung der
Reichweite des hier besprochenen Urteils führen, da diese nicht auf sämtliche Behandler im Screening übertragbar sind. Gleichzeitig können sie bei der Fehlervermeidung helfen, sofern sie nicht bereits berücksichtigt werden. Zunächst sollte bei Durchführung des Screenings sichergestellt sein, dass die Radiologen die jeweilige Anamnese der Patientin zur Kenntnis nehmen. Sollten sich hieraus Verdachtsmomente ergeben, ist zumindest ein entsprechender Hinweis an die Patientin vorzunehmen. Ein positives Abschlussschreiben sollte auch bei unauffälliger Mammographie nicht ohne Vorbehalt formuliert sein, sondern in jedem Fall auf die begrenzte Aussagekraft des Screening-Befundes hinweisen. Zu vermeiden ist der Eindruck eines zweifelsfreien und vollumfänglich unauffälligen Befundes für den aktuellen Status, also - missverständlich - auch mit Bedeutung über das Screening hinaus.
Prof. Dr. Peter Wigge

Rechtsanwalt

Fachanwalt für Medizinrecht

Tilmann Kirsch

Rechtsanwalt

Rechtsanwälte Wigge

Großer Burstah 42

20457 Hamburg

Tel.: (040) 3398 705-90

Fax: (040) 3398 705-99

Internet: www.ra-wigge.de

E-Mail: kanzlei@ra-wigge.de 